\title{
Optical fiber multiplexing interferometer system with a single laser diode and its application to online displacement measurement
}

\author{
Fang Xie \\ Yi Lu \\ Beijing Jiaotong University \\ School of Science \\ Department of Physics \\ Optical Science and Technology Laboratory \\ Beijing 100044, China \\ E-mail: fxie@ @jtu.edu.cn \\ Lin Zhang \\ Aston University \\ School of Engineering and Applied Science \\ Photonics Research Group \\ Birmingham, B4 7ET, United Kingdom
}

\begin{abstract}
A multiplexed optical fiber Michelson interferometer system that is self-referenced with a stabilizing feedback loop is presented. This system employs fiber Bragg gratings and wavelength division multiplexing technique to combine two optical fiber interferometers that share the same optical path in the main part of the optical system. When one Michelson interferometer, which uses the fiber Bragg gratings as reflective mirrors and is used as reference interferometer, is stabilized by an electric feedback loop, the other interferometer, which is used for the measurement, is also stabilized. This system is therefore suitable for online precision measurement. An active phase-tracking technique is applied for signal processing to achieve high resolution. () 2007 Society of Photo-Optical Instrumentation Engineers. [DOI: 10.1117/1.2738723]
\end{abstract}

Subject terms: metrology; fiber optics; interferometry; measurement; displacement.

Paper 060854SSRR received Oct. 31, 2006; revised manuscript received Mar. 15, 2007; accepted for publication Mar. 15, 2007; published online May 22, 2007.

\section{Introduction}

Interferometers have been employed to measure precision displacement because of their high measurement resolution. ${ }^{1-9}$ Optical fiber interferometers have been widely used for online precision measurement due to their prominent advantages such as noncontact, compactness, light weight, high resolution, and low cost. ${ }^{1-4}$ There are also many needs for online precision measurement in the transportation industry. The optical fiber interferometer is very suitable for that purpose. Besides being sensitive to information signals, however, the optical fiber interferometer is also sensitive to noise resulting from temperature fluctuations and other types of disturbances. Thus, optical fiber interferometers should be stabilized to remove the noise in order to obtain high measurement accuracy when used for online measurements in the presence of temperature fluctuations and other types of environmental noise. ${ }^{5}$

The system described in Ref. 5 used an electric feedback loop driving a piezoelectric tube wound with the fiber used in the reference arm to compensate for the error induced by the environmental noises. A high-pass electric filter connected to the feedback loop removes noise from the signal information. The information must be an alternating signal: otherwise it cannot be distinguished from the noise. In Ref. 9, we reported the basic idea of stabilizing a multiplexed optical fiber interferometer and its application to precision measurement. ${ }^{9}$ The displacement measurement, however, would be interrupted when a voltage reset action on the PZT1 tube occurred. This meant that the displacement measured after voltage reset would not be continuous with the displacement measured before. The process of measurement had to be finished before any voltage reset action

0091-3286/2007/\$25.00 @ 2007 SPIE occurred. The frequency of the voltage reset action in the experiments was about $0.2 \mathrm{~Hz}$ in the lab environment, so it was difficult to use the system for online measurement.

The system presented in this paper has greatly developed the idea described in Ref. 9. Based on the characteristics of fiber Bragg gratings (FBGs) and wavelength division multiplexing technique, the system includes two optical fiber Michelson interferometers, which share the same optical path in the main part of the optical system. The overlapped interferometers are probed respectively by different parts of the spectrum of a single laser diode (LD), with full width at half maximum (FWHM) $1.5 \mathrm{~nm}$. One of the interferometers is used as a reference to monitor the noise and is compensated for with an electric feedback loop, while the other is used to do the measurement. Two FBGs of the same characteristic (the FWHM of the reflected Bragg spectrum is $0.1 \mathrm{~nm}$ ) are incorporated into the two interference arms and are used as reflective mirrors in the reference interferometer. A piezoelectric tube wound with the fiber in one arm of the interferometer is driven by an electrical feedback loop to tune the length of the arm and keep the phase difference between the two arms at $\pi / 2 \mathrm{rad}$ at all times. The stabilization of the reference interferometer is realized in this way. Because it shares the same optical path in the main part of the optical system with the measurement interferometer, the measurement interferometer is also thereby stabilized. The FWHM of the reflected Bragg wavelengths of the two FBGs is $0.1 \mathrm{~nm}$, and the corresponding coherence length is $24 \mathrm{~mm}$, so the reference Michelson interferometer is easy to configure.

Although sharing the main optical path of the optical system, the reflective mirrors of the measurement interferometer and those of the reference interferometer are independent. The two multiplexed interferometers are probed by a single laser diode. This prevents voltage reset from 


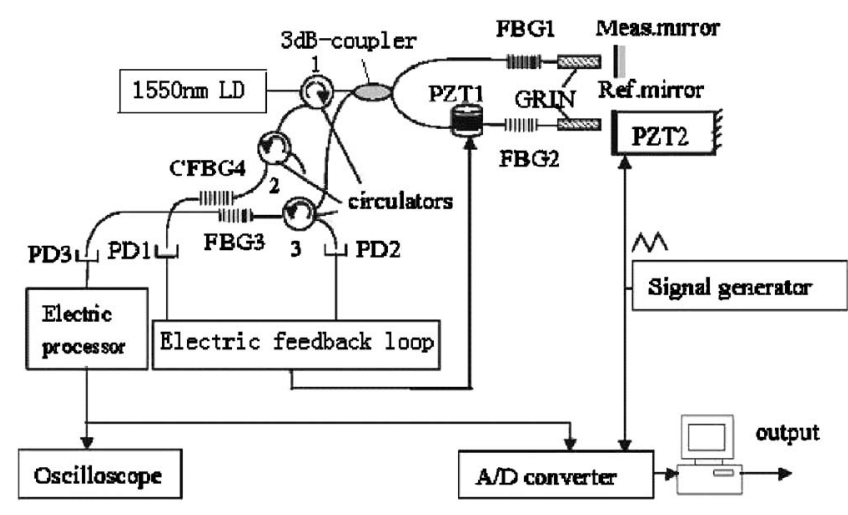

Fig. 1 The principle of the system.

interrupting the displacement measurement. The measurement action can be continuous whether there is voltage reset or not. The measured signal can be alternating or static information. We here concentrate on studying the measurement of static absolute displacement. On applying active phase tracking, the displacement measurement resolution of the system can reach $2 \mathrm{~nm}$.

\section{System and Experimental Results}

\subsection{Principle of the System}

The multiplexed fiber interferometer combines two fiber Michelson interferometers, which share the main optical path of the optical system, as is shown in Fig. 1. A laser diode with output power $1.5 \mathrm{~mW}$, center wavelength at $1550 \mathrm{~nm}$, and spectral FWHM $1.5 \mathrm{~nm}$ is used to probe the two fiber interferometers. Three FBGs, one chirped, are incorporated into the optical system. FBG1, FBG2, and FBG3 have the same parameters. The reflected Bragg wavelength of the FBGs is addressed at $1549.5 \mathrm{~nm}$ with spectral FWHM $0.1 \mathrm{~nm}$, while the chirped FBG4 will reflect light with wavelength between 1549.6 and $1552 \mathrm{~nm}$.

The first fiber Michelson interferometer, which employs FBG1 and FBG2 as the reflective mirrors in the two arms, is used as a reference interferometer to monitor and compensate for the differential phase drift in the two arms induced by environmental noise. The light emitted from the 1550-nm LD passes through circulator 1 and the $3-\mathrm{dB}$ coupler, and then is divided into two beams. Parts of the spectra of the two beams are reflected back by FBG1 and FBG2, respectively, and combine again at the $3-\mathrm{dB}$ coupler. The interferometric light from one output of the coupler passes through circulator 1 , circulator 2 , and the chirped FBG4 and is received by photodetector 1 (PD1), while the interferometric light from the other output of the coupler passes through circulator 3, is reflected by FBG3 (so it will not reach PD3), and is received by photodetector 2 (PD2). The interferometric signals detected by PD1 and PD2 have a phase difference of $\pi \mathrm{rad}$. The signals from PD1 and PD2 are processed electrically by the feedback loop, and the output of the loop is applied to the piezoelectric tube PZT1 (PI product, PIS-C, length $25 \mathrm{~cm}$, outer diameter $2.5 \mathrm{~cm}$, wall thickness $0.2 \mathrm{~cm}$ ) wound with approximately $9 \mathrm{~m}$ of the fiber used in the reference arm. The signal applied to this tube is the correction signal required to keep the phase difference between the interferometer arms at $\pi / 2 \mathrm{rad}$. It

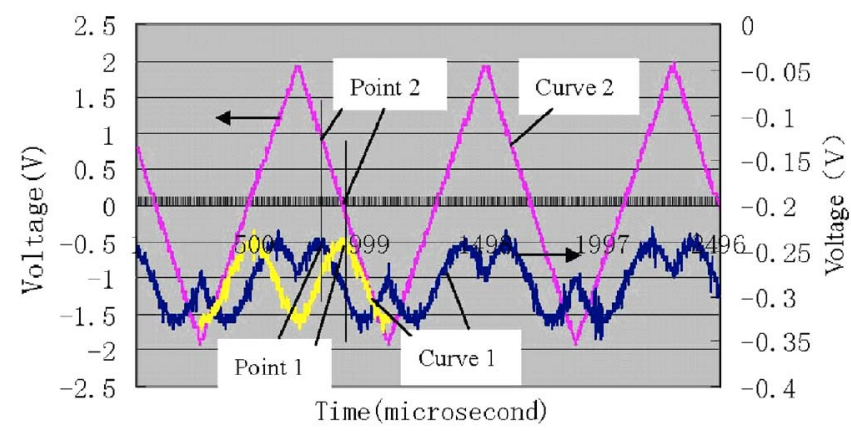

Fig. 2 The voltage modulation and the detected signal at PD3.

contains information on the drift and noise resulting from temperature fluctuations and other types of disturbances. The reference interferometer is locked in quadrature in this way. The influence of environmental noise is effectively eliminated.

The second Michelson interferometer is probed by the other part of the spectrum from the LD, which obtains displacement information from the measurement mirror. Because FBG1 and FBG2 are placed respectively just before the collimator gradient-index (GRIN) lenses, the measurement interferometer shares almost its whole optical path with the reference interferometer. The disturbances from the environment to the measurement interferometer will also be eliminated when the reference interferometer is locked.

The parts of the spectra of the two beams that are not reflected back by FBG1 and FBG2 pass through the collimator GRIN lenses and then are reflected back by the measurement mirror and the reference mirror, respectively. The two reflected beams combine at the 3-dB coupler. The interferometric light from one output of the coupler passes through circulator 3 and FBG3 and is received by photodetector 3 (PD3). The interferometric light from the other output of the coupler goes through circulators 1 and 2 and is reflected back by the chirped FBG4; it does not reach PD1. By employing the characteristics of FBGs and wavelength division multiplexing, the system effectively separates the measurement signal from the drift and noise signals.

A voltage change is applied to PZT2 to modulate the optical path of the reference arm of the measurement interferometer. The interference signal detected by PD3 will vary corresponding, as is shown in Fig. 2. Curve 1 is the interferometric signal detected by PD3, while curve 2 is the voltage modulation applied to PZT2. We tune the amplitude of the voltage change to make it correspond to a phase change of $2 \pi \mathrm{rad}$ in the interferometric signal while the voltage modulation is driving PZT2. When the displacement changes, the peak of curve 1 during half a period of the voltage modulation will shift linearly. Curve 1 and curve 2 are converted simultaneously into digital signals by an analog-to-digital (A/D) converter and are processed in a personal computer. The shift of the peak on curve 1 will be determined easily by the software, and the difference between the two values on curve 2 that correspond to the peak on curve 1 before and after the shift will also be measured, 


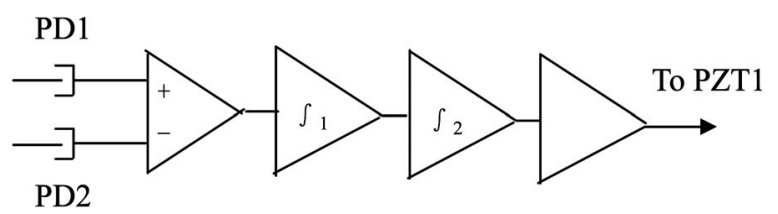

Fig. 3 The block diagram of the feedback stabilization circuit.

as shown in Fig. 2. The phase change in the interferometric signal induced by the displacement can therefore be calculated.

\subsection{Block Diagram of the Feedback Stabilization Circuit}

The block diagram of the feedback stabilization circuit is shown in Fig. 3. The interferometric signals of the reference interferometer from the two outputs of the 3-dB coupler are always complementary to each other, whether the stabilizing circuit is turned on or off. The signals received by PD1 and PD2 have a phase difference of $\pi$ rad. The current outputs from PD1 and PD2, $i_{1}$ and $i_{2}$, which correspond to the output intensities $I_{1}$ and $I_{2}$, will therefore have the form

$i_{1}=i_{0}\left[1-k \cos \left(\phi_{d}+\phi_{s}\right)\right]$

$i_{2}=i_{0}\left[1+k \cos \left(\phi_{d}+\phi_{s}\right)\right]$,

where $i_{0}$ is related to the input optical power, $k$ is a function of the output fringe visibility, $\phi_{d}$ is the static differential phase between the two arms, and $\phi_{s}$ is the phase induced by the perturbation. Differentially combining these photocurrents with an electrical subtraction circuit produces an output

$i_{3}=2 i_{0} k \cos \left(\phi_{d}+\phi_{s}\right)$.

The signal of Eq. (3) is integrated twice by two integrators. An amplifier is connected to the second integrator to amplify its output about 100 times. The amplifier's output is applied to the piezoelectric tube and drives it to track the phase difference between the two arms. When $\phi_{d}+\phi_{s}$ $=\pi / 2$, the feedback voltage is zero and the two arms are in quadrature; when $\phi_{d}+\phi_{s} \neq \pi / 2$, the feedback voltage drives the piezoelectric tube PZT1 to tune the length of the fiber and put the two arms in quadrature again.

With $9 \mathrm{~m}$ of fiber wound on the piezoelectric tube, the circuit can continuously track differential phase differences of up to about $\pm 10 \pi$ rad. Should the phase difference become larger than $10 \pi$ rad, a fast-responding circuit resets the voltage applied to the piezoelectric tube, so that the voltage after resetting is close to zero. The tracking action can be continuous due to the resetting circuit. The voltage reset action does not interrupt the displacement measurement. The reason is explained below.

A single laser diode with a spectral FWHM of $1.5 \mathrm{~nm}$ is used to probe both the measurement interferometer and the reference interferometer. The FWHM of the Bragg wavelength of the two FBGs in the arms is $0.1 \mathrm{~nm}$, and they reflect some part of the spectrum of the LD. The other part of the spectrum of the LD is used to probe the measurement

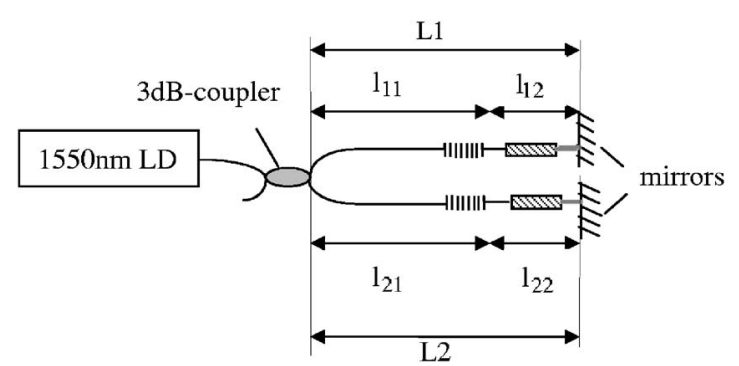

Fig. 4 Explanation of continuous measurement.

interferometer. This is explained in Fig. 4. The lengths of the two arms of the measurement interferometer are

$L_{1}=l_{11}+l_{12}$

$L_{2}=l_{21}+l_{22}$.

The optical path difference of the measurement interferometer is

$\Delta_{m}=L_{2}-L_{1}=\left(l_{21}-l_{11}\right)+\left(l_{22}-l_{12}\right)$.

The phase difference is

$$
\begin{aligned}
\Delta \varphi_{m} & =\frac{\Delta_{m}}{\lambda} 2 \pi \\
& =\frac{l_{21}-l_{11}}{\lambda} 2 \pi-\frac{l_{22}-l_{12}}{\lambda} 2 \pi \\
& =\Delta \varphi_{r}-\Delta \varphi \\
& =(2 n+1) \frac{\pi}{2}-\Delta \varphi .
\end{aligned}
$$

From Eq. (5), it is seen that the effective value of $\Delta \varphi_{m}$ is determined by the second term and is not related to the optical path difference of the reference interferometer. This makes the displacement measurement always continuous, whether a voltage reset has occurred or not.

\subsection{Experimental Results}

The environmental disturbances, such as vibration and drift of temperature, seriously affect the stability of the fiber interferometer. However, the disturbances are successfully compensated for by the feedback system of the multiplexing optical fiber Michelson interferometer. The stabilizing effect is shown in Fig. 5. The output of PD3 is fluctuating frequently from 0 to $300 \mathrm{mV}$ before stabilization, but with the feedback loop circuit turned on, the output of PD3 maintains almost a constant value, with a fluctuation amplitude of about $3 \mathrm{mV}$, which is induced by the noise of electrical processing. The measurement interferometer is effectively stabilized and can be used for online precision measurement.

The amplitude of the voltage modulation applied to PZT2 is tuned to $2 \mathrm{~V}$, which corresponds to a $2 \pi$-rad phase change in the interferometric signal of the measurement interferometer. The measurement accuracy is determined by the performance of PZT2, the A/D converter, and the elec- 


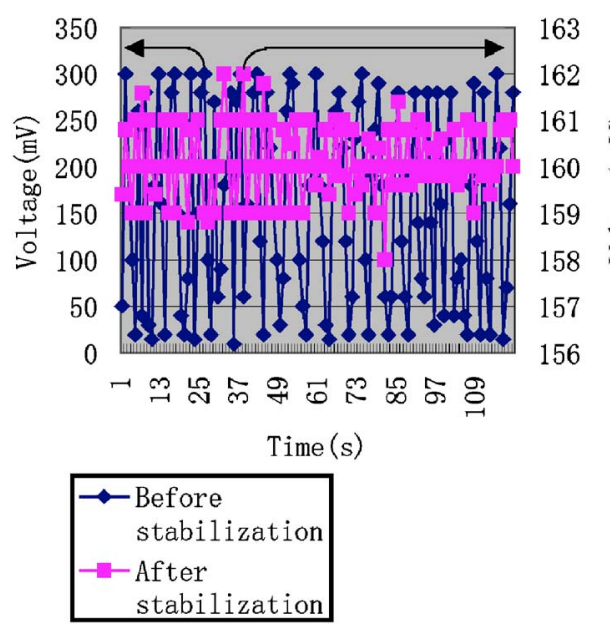

Fig. 5 The output of PD3 before and after stabilization.

tric feedback loop. The resolution of PZT2 (PI product, $\mathrm{P}-882$ ) is subnanometer, and its performance linearity is $99 \%$. The A/D converter output is 12 bits. The amplitude of the input voltage of $\mathrm{A} / \mathrm{D}$ converter is $10 \mathrm{~V}$, and its voltage resolution is $(10 \mathrm{~V}) / 2^{12}=2.4 \mathrm{mV}$. The corresponding minimum phase change that can be measured is $\Delta \theta_{\text {min }}$ $=[(360 \mathrm{deg}) /(2000 \mathrm{mV})] \times 2.4 \mathrm{mV}=0.4 \mathrm{deg}$. Because the wavelength of the light source is $1550 \mathrm{~nm}$, the corresponding minimum displacement that the $\mathrm{A} / \mathrm{D}$ converter can resolve is

$\Delta d=\frac{\Delta \theta_{\min } \lambda}{2 \times(360 \mathrm{deg})}=\frac{(0.4 \mathrm{deg}) \times 1550 \mathrm{~nm}}{2 \times(360 \mathrm{deg})} \approx 0.9 \mathrm{~nm}$.

Considering the error of electrical processing in the feedback loop, the limited resolution of the A/D converter, and the performance of PZT2, the resolution of the system can reach $2 \mathrm{~nm}$. A measured result over a $6-\mu \mathrm{m}$ range is given in Fig. 6, in which it can be seen that the points are almost on a line, with very little scatter. After linear fitting of the experiment data by the least-squares method, it is obtained

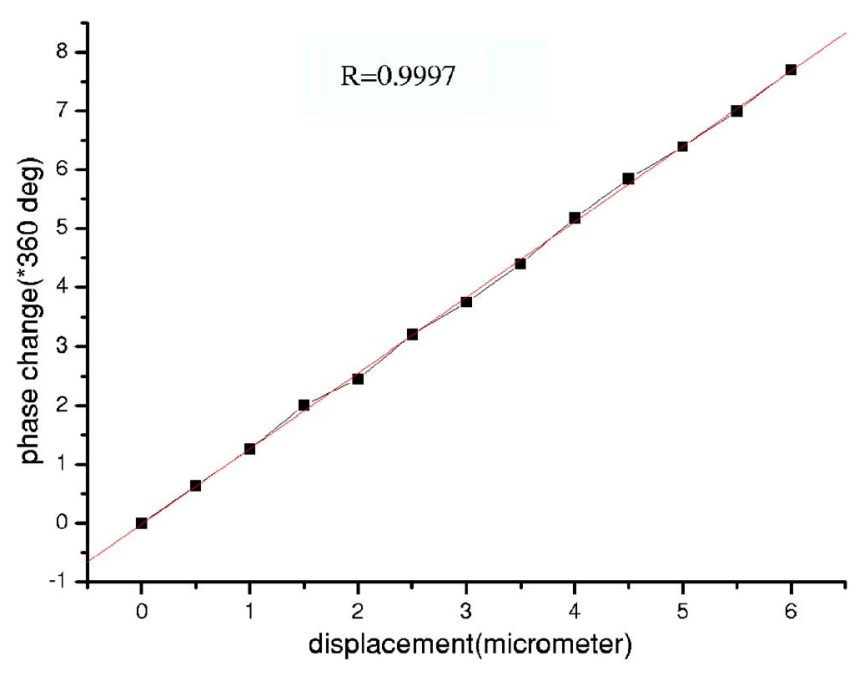

Fig. 6 The experimental results. that the corresponding linear regression coefficient is 0.9997, which implies that the system has good linearity. And this linear response indicates a fixed relation between the displacement and the phase change in the interferometric signal, viz., $\lambda / 2$ against $2 \pi$.

\section{Conclusion}

A stable multiplexing fiber Michelson interferometer system incorporating fiber Bragg gratings, which is probed with a single LD, has been presented. Two fiber Michelson interferometers, respectively acting as a measurement interferometer and a reference interferometer, share the main optical path in the system. An electrical feedback loop drives the piezoelectric tube wound with the fiber in the reference arm to lock the phase difference between two interferometer arms at $\pi / 2$. The system is stabilized and is suitable for online measurement. An active phase-tracking technique is applied. The displacement measurement resolution of the system can reach $2 \mathrm{~nm}$.

\section{Acknowledgments}

This research is supported by Beijing Natural Science Foundation and National Natural Science Foundation of China.

\section{References}

1. D. P. Hand, T. A. Carolan, J. S. Barton, and J. D. C. Jones, "Profile measurement of optically rough surfaces by fiber-optic interferometry," Opt. Lett. 18, 1361-1363 (1997).

2. D. A. Jackson, A. Dandridge, and S. K. Sheem, "Measurements of small phase shifts using a single-mode optical fiber interferometer," Opt. Lett. 5, 139-141 (1980).

3. D. A. Jackson, A. Dandridge, and A. B. Tventen, "Elimination of drift in a single-mode optical fiber interferometer using a piezoelectric stretched coiled fiber," Appl. Opt. 19, 2926-2929 (1980).

4. L. Delage and F. Reynaud, "Kilometric optical fiber interferometer," Opt. Express 9(6), 267-271 (2001).

5. K. Fritsch and G. Adamovsky, "Simple circuit for feedback stabilization of a single-mode optical fiber interferometer," Rev. Sci. Instrum. 52, 996-1000 (1981).

6. N. Bobroff, "Recent advances in displacement measuring interferometry," Meas. Sci. Technol. 4, 907-926 (1993).

7. K. J. Scott, Three-Dimensional Surface Tomography: Measurement, Interpretation, and Application, Jessica Kingsley Publishers Ltd., London (1994).

8. Y. Zhou, T. Zhou, and D. Li, "Heterodyne absolute distance interferometer with a dual-mode He-Ne laser," Opt. Eng. 38, 246-249 (1999).

9. D. Lin, X. Jiang, F. Xie, W. Zhang, L. Zhang, and I. Bennion, "High stability multiplexed fiber interferometer and its application on absolute displacement measurement and on-line surface metrology," Opt. Express 12(23), 5729-5734 (2004).

Fang Xie received her BSc (mechanical engineering) at Chongqing University, China, in 1987; received her MSc (optics and precision instruments) at Chongqing University, China, in 1992; and received her PhD (optical engineering) at Tsinghua University, China, in 2002. She was funded by Huddersfield University and worked as a visiting research fellow at Aston University in 2002-2003. She is now an associate professor in the Physics Department at Beijing Jiaotong University, China. She has authored and coauthored more than thirty research papers in journals and at conferences. Her research interest is in the fields of optical fiber sensors, and optical precision measurement and instruments.

Yi Lu received his BSc at Beijing Jiaotong University, China, in 2004. He is now a postgraduate student in the Physics Department, Beijing Jiaotong University, China. 
Xie, Lu, and Zhang: Optical fiber multiplexing interferometers system...

Lin Zhang received her BSc at Suzhou University, China, in 1978; received her MSc at Suzhou University, China, in 1986; and received her PhD at Sussex University, UK, in 1991. She is member of the IOP, OSA, and IEEE. She was a research fellow at Sussex University in 1990-1994, was a research fellow at Aston University, UK, in 1994-1999, and was a lecturer at Aston University in 20002005. She is now a professor at Aston University. She has published more than 300 papers in international standard journals and conferences. Her research interest is in novel photonic devices and their applications in telecommunications, optical fiber sensing, and biophotonics. 